\title{
Regularized cosine existence and uniqueness families for second order abstract Cauchy problems
}

by

\author{
JizhOU ZHANG (Shanghai)
}

\begin{abstract}
Let $\mathbf{C}_{i}(i=1,2)$ be two arbitrary bounded operators on a Banach space. We study $\left(\mathbf{C}_{1}, \mathbf{C}_{2}\right)$-regularized cosine existence and uniqueness families and their relationship to second order abstract Cauchy problems. We also prove some of their basic properties. In addition, Hille-Yosida type sufficient conditions are given for the exponentially bounded case.
\end{abstract}

Introduction. Let $X$ be a Banach space with norm $\|\cdot\|$, and $B(X)$ be the set of all bounded operators from $X$ into itself. Consider a well-posed abstract Cauchy problem of the second order

$$
\left\{\begin{array}{l}
\frac{d^{2}}{d t^{2}} u(t, x, y)=A u(t, x, y), \quad t \in \mathbb{R}, \\
u(0, x, y)=x, \quad \frac{d}{d t} u(0, x, y)=y,
\end{array}\right.
$$

or

$$
\left\{\begin{array}{l}
\frac{d^{2}}{d t^{2}} v(t, x, y)=A v(t, x, y)+x+t y, \quad t \in \mathbb{R}, \\
v(0, x, y)=0, \quad \frac{d}{d t} v(0, x, y)=0
\end{array}\right.
$$

where $A$ is a closed linear operator on $X$. It is well known that $(0.1)$ or $(0.2)$ is governed by a strongly continuous operator cosine function (see [12, 13]). Cosine families have received less attention than strongly continuous semigroups, partly because (0.1) may be rewritten as a first order abstract

2000 Mathematics Subject Classification: Primary 47D06, 47D09; Secondary 34G10.

Key words and phrases: regularized cosine existence and uniqueness family, regularized cosine function, Cauchy problem.

This project is supported by the Natural Science Foundation of China and Shanghai City. 
Cauchy problem

$$
\left\{\begin{array}{l}
\frac{d}{d t} u(t, x)=\left[\begin{array}{ll}
0 & 1 \\
A & 0
\end{array}\right] u(t, x) \quad(t \geq 0), \\
u(0, x)=x
\end{array}\right.
$$

so that one may discuss semigroups generated by $\left[\begin{array}{ll}0 & 1 \\ A & 0\end{array}\right]$. However, this matrix reduction is not always successful; it is sometimes necessary to leave (0.1) as a second order problem and sometimes it is simpler to work with a cosine family generated by $A$ rather than the semigroup generated by $\left[\begin{array}{ll}0 & 1 \\ A & 0\end{array}\right]$. We know that many partial differential operators in $L^{p}\left(\mathbb{R}^{n}\right)$ such as the Laplace operator $\Delta$ with maximal distributional domain do not generate a strongly continuous cosine function unless $n=1,1 \leq p<\infty$ or $n \geq 2$ and $p=2$ (see [8]). Recently, two new cosine functions of operators, i.e., regularized cosine functions (see $[3,4,11,16])$ and integrated cosine functions (see $[2$, $10,15,18]$ ) have been extensively studied. The goal of these generalizations is to apply operator theory to second order Cauchy problems where unique solutions exist for a nontrivial set of initial data which is not equal to the entire domain of $A$. Numerous examples where regularized or integrated cosine functions may be applied directly, but strongly continuous cosine functions may not, appear in $[2-4,10,16]$. However, there are limitations to both regularized and integrated cosine functions. There exist operators which generate neither a regularized nor an integrated semigroup (see [5-7, 14]). Likewise, there exist operators which generate neither a regularized nor an integrated cosine function (see [15] and Example 3.1 below).

A good generalization of strongly continuous cosine functions should satisfy the following. When unique solutions of $(0.1)$ or $(0.2)$ exist for a nontrivial set of initial data, they should be accessible through this family of operators, without any renorming or constructions of new Banach spaces. On the other hand, it should bring benefits analogous to those of strongly continuous cosine functions.

The motivation for this paper comes from deLaubenfels' paper [5] where a pair of families of operators is defined, one of which yields uniqueness, while the other yields existence of solutions of the first order abstract Cauchy problem. In this paper, we also present a pair of families of operators that we believe will have the desired properties mentioned in the previous paragraph, a $\mathbf{C}_{1}$-existence family, which yields a solution of (0.2) for all $x$ in $\mathcal{R}\left(\mathbf{C}_{1}\right)$, and a $\mathbf{C}_{2}$-uniqueness family, which yields uniqueness of any solutions of $(0.1)$ or (0.2) (see Definitions 1.1, 1.3-1.5). The operator $\mathbf{C}_{2}$ must be bounded and injective, the only requirement on $\mathbf{C}_{1}$ is that it be bounded. Intuitively, the $\mathbf{C}_{1}$-regularized cosine existence family is $\cosh (t \sqrt{A}) \mathbf{C}_{1}$ and the $\mathbf{C}_{2^{-}}$ regularized cosine uniqueness family is $\mathbf{C}_{2} \cosh (t \sqrt{A})$. When $\mathbf{C}_{1}=\mathbf{C}_{2}$, and 
commutes with $A$, then $\cosh (t \sqrt{A}) \mathbf{C}_{1}=\mathbf{C}_{2} \cosh (t \sqrt{A})$ is a $\mathbf{C}_{2}$-regularized cosine function.

The paper is organized as follows. We first give in Section 1 the definition of $\left(\mathbf{C}_{1}, \mathbf{C}_{2}\right)$-regularized cosine existence and uniqueness families, and their relationship to (0.1) and (0.2). We also prove some of their basic properties. In Section 2 we obtain Hille-Yosida type sufficient conditions for generating exponentially bounded $\mathbf{C}_{1}$-regularized cosine existence families. Finally, we give two examples in Section 3.

\section{1. $\left(\mathbf{C}_{1}, \mathbf{C}_{2}\right)$-regularized cosine families and their basic proper-} ties. We shall write $\mathcal{D}(A)$ for the domain of the linear operator $A, \mathcal{R}(A)$ for its range and $R(\lambda, A)$ for its resolvent. In the following, we first give the definition of a $\left(\mathbf{C}_{1}, \mathbf{C}_{2}\right)$-regularized cosine existence and uniqueness family $\left\{C_{1}(t), C_{2}(t)\right\}_{t \in \mathbb{R}}$. Then we study the basic properties of the generator and the relationship with (0.1) or (0.2) as well as the Laplace transform.

Definition 1.1. A strongly continuous family $\left\{C_{1}(t), C_{2}(t)\right\}_{t \in \mathbb{R}}$ of pairs of bounded operators on $X$ is called a mild $\left(\mathbf{C}_{1}, \mathbf{C}_{2}\right)$-regularized cosine existence and uniqueness family on $X$ if

(a) $C_{i}(0)=\mathbf{C}_{i}$ for $i=1,2$;

(b) $\mathbf{C}_{2}$ is injective;

(c) $2 C_{2}(t) C_{1}(s)=\mathbf{C}_{2}\left[C_{1}(t+s)+C_{1}(t-s)\right]$

$$
=\left[C_{2}(t+s)+C_{2}(t-s)\right] \mathbf{C}_{1}, \quad \forall t, s \in \mathbb{R} .
$$

Since $\mathbf{C}_{2}$ is injective, one can define the generator $A$ of the family $\left\{C_{1}(t), C_{2}(t)\right\}_{t \in \mathbb{R}}$ by

$$
\left\{\begin{array}{l}
A x=\mathbf{C}_{2}^{-1} C_{2}^{\prime \prime}(0) x \\
\mathcal{D}(A)=\left\{x \in X \mid C_{2}^{\prime \prime}(t) x\right. \text { exists, and } \\
\left.\quad \text { is equal to } C_{2}(t) \mathbf{C}_{2}^{-1} C_{2}^{\prime \prime}(0) x, \forall t \in \mathbb{R}\right\}
\end{array}\right.
$$

or equivalently

$$
\left\{\begin{array}{l}
A x=\mathbf{C}_{2}^{-1} \lim _{h \rightarrow 0} \frac{2}{h^{2}}\left\{C_{2}(h) x-\mathbf{C}_{2} x\right\}, \\
\mathcal{D}(A)=\left\{x \in X \mid \text { the limit exists and is in } \mathcal{R}\left(\mathbf{C}_{2}\right)\right\} .
\end{array}\right.
$$

REMARK 1.2. It is obvious that $C_{1}(t)$ is equal to $C_{2}(t)$ when $C_{1}(t)$ is a $\mathbf{C}_{1}$-regularized cosine function, and $A$ is its generator when $\mathbf{C}_{1}$ is equal to $\mathbf{C}_{2}$ and commutes with $C_{1}(t)$ and $C_{2}(t)$ for all $t \in \mathbb{R}$. Intuitively, we may write that $C_{1}(t)=\cosh (t \sqrt{A}) \mathbf{C}_{1}, C_{2}(t)=\mathbf{C}_{2} \cosh (t \sqrt{A})$, respectively.

In the following, we will denote the $\mathbf{C}_{1}$-resolvent set of $A$ by

$$
\varrho_{\mathbf{C}_{1}}(A)=\left\{\lambda \mid \lambda-A \text { is injective and } \mathcal{R}\left(\mathbf{C}_{1}\right) \subseteq \mathcal{R}(\lambda-A)\right\}
$$


and

$$
\varrho_{\mathbf{C}_{1}}(\bar{A})=\left\{\lambda \mid \lambda-\bar{A} \text { is injective and } \mathcal{R}\left(\mathbf{C}_{1}\right) \subseteq \mathcal{R}(\lambda-A)\right\} .
$$

Definition 1.3. Suppose $A$ is closable. A strongly continuous family $\left\{C_{1}(t)\right\}_{t \in \mathbb{R}} \subset B(X)$ of operators is an exponentially bounded (resp. mild) $\mathbf{C}_{1}$-regularized cosine existence family for $A$ if $t \mapsto C_{1}(t) x \in C(\mathbb{R},[\mathcal{D}(A)])$ $\left(\right.$ resp. $\left.\int_{0}^{t}(t-s) C_{1}(s) x d s \in \mathcal{D}(A), t \mapsto A\left(\int_{0}^{t}(t-s) C_{1}(s) x d s\right) \in C(\mathbb{R}, X)\right)$ for all $x \in \mathcal{D}(A)$ (resp. $x \in X$ ) and $t \in \mathbb{R}$, and there exist $M, \omega>0$ such that $\left(\omega^{2}, \infty\right) \subseteq \varrho_{\mathbf{C}_{1}}(\bar{A}),\left\|A C_{1}(t)\right\|\left(\right.$ resp. $\left.\left\|C_{1}(t)\right\|\right) \leq M e^{\omega|t|}$, and

$$
R\left(\lambda^{2}, A\right) \mathbf{C}_{1} x=\lambda^{-1} \int_{0}^{\infty} e^{-\lambda t} C_{1}(t) x d t \quad \text { for all } x \in X \text { and } \lambda>\omega .
$$

Definition 1.4. A strongly continuous family $\left\{C_{2}(t)\right\}_{t \in \mathbb{R}} \subset B(X)$ of operators is an exponentially bounded $\mathbf{C}_{2}$-regularized cosine uniqueness fam$i l y$ for $A$ if $\mathbf{C}_{2}$ is injective and there exist $M, \omega>0$ such that $\left\|C_{2}(t)\right\| \leq$ $M e^{\omega|t|}$ for all $t \in \mathbb{R}$ and

$$
\mathbf{C}_{2} x=\lambda^{-1} \int_{0}^{\infty} e^{-\lambda t} C_{2}(t)\left(\lambda^{2}-A\right) x d t \quad \text { for all } x \in \mathcal{D}(A) \text { and } \lambda>\omega .
$$

Definition 1.5. Suppose that $\mathbf{C}_{1} \in B(X)$.

(a) We shall say that (0.1) is $\mathbf{C}_{1}$-well-posed if it has a unique solution $u$ for every pair $(x, y) \in \mathbf{C}_{1} \mathcal{D}(A) \times \mathbf{C}_{1} \mathcal{D}(A)$ and there exists a continuous $g: \mathbb{R} \rightarrow \mathbb{R}$ such that

$$
\left\|u\left(t, \mathbf{C}_{1} x, \mathbf{C}_{1} y\right)\right\| \leq g(t)(\|x\|+|t| \cdot\|y\|) \quad \text { for all } t \in \mathbb{R} .
$$

(b) We shall say that (0.2) is $\mathbf{C}_{1}$-well-posed if it has a unique solution $v$ for all $x, y \in \mathcal{R}\left(\mathbf{C}_{1}\right)$, and there exists a continuous $g: \mathbb{R} \rightarrow \mathbb{R}$ such that

$$
\left\|\frac{d^{2} v}{d t^{2}}\left(t, \mathbf{C}_{1} x, \mathbf{C}_{1} y\right)\right\| \leq g(t)(\|x\|+|t| \cdot\|y\|) \quad \text { for all } t \in \mathbb{R} .
$$

(c) (0.1) (or (0.2)) is called $\mathbf{C}_{1}$-exponentially well-posed if there exist a unique exponentially bounded solution of (0.1) (or (0.2)) and $M, \omega>0$ such that $g(t)=M e^{\omega|t|}$ for all $t \in \mathbb{R}$ in (a) (or (b)).

We start with some basic properties of $\left(\mathbf{C}_{1}, \mathbf{C}_{2}\right)$-regularized cosine families.

Theorem 1.6. Suppose that $A$ generates a $\left(\mathbf{C}_{1}, \mathbf{C}_{2}\right)$-regularized cosine family $\left\{C_{1}(t), C_{2}(t)\right\}_{t \in \mathbb{R}}$. Then

(a) $A$ is closed;

(b) $C_{1}(t)=C_{1}(-t)$; 
(c) $\int_{0}^{t}(t-s) C_{1}(s) x d s \in \mathcal{D}(A)$ for all $t \in \mathbb{R}$ and $x \in X$, and

$$
A \int_{0}^{t}(t-s) C_{1}(s) x d s=C_{1}(t) x-\mathbf{C}_{1} x
$$

(d) $\mathcal{R}\left(\mathbf{C}_{1}\right) \subset \overline{\mathcal{D}(A)}$;

(e) if $\left\|C_{i}(t)\right\| \leq M e^{\omega|t|}$ for some $\omega>0$ and $t \in \mathbb{R}, i=1,2$, then $\left(\omega^{2}, \infty\right) \subset \varrho \mathbf{C}_{1}(A)$ and $(1.2)$ and $(1.3)$ hold.

Proof. (a) By (1.1), we have

$$
C_{2}^{\prime \prime}(t) x=C_{2}(t) A x \quad \text { for } x \in \mathcal{D}(A) \text { and } t \in \mathbb{R} .
$$

Let $\left\{x_{n}\right\}_{n=1}^{\infty} \subset \mathcal{D}(A), x_{n} \rightarrow x$ and $A x_{n} \rightarrow y$ as $n \rightarrow \infty$. Then $C_{2}(t) x_{n}$ converges to $C_{2}(t) x$ and $C_{2}(t) A x_{n}$ converges to $C_{2}(t) y$ uniformly on compact sets. The strong continuity of $C_{2}(t)$ now implies that $C_{2}^{\prime \prime}(t) x$ exists, and is equal to $C_{2}(t) y$ for all $t \in \mathbb{R}$. This implies that $x \in \mathcal{D}(A)$ and therefore $A x=y$, as desired.

(b) follows from Definition 1.1(c).

(c) Let $y=\int_{0}^{t}(t-s) C_{1}(s) x d s$ for all $x \in X$ and $t \in \mathbb{R}$. Then, for any $h>0$, we have

$$
\begin{aligned}
2 h^{-2}\left(C_{2}(h) y-\mathbf{C}_{2} y\right)= & \mathbf{C}_{2} h^{-2} \int_{0}^{t}(t-s)\left(C_{1}(s+h)+C_{1}(s-h)-2 C_{1}(s)\right) x d s \\
= & \mathbf{C}_{2}\left[h^{-1}\left(\int_{h}^{t+h}-\int_{-h}^{t-h}\right) C_{1}(s) x d s\right. \\
& +h^{-2}\left(\int_{t}^{t+h}+\int_{t}^{t-h}\right)(t-s) C_{1}(s) x d s \\
& \left.-h^{-2}\left(\int_{0}^{h}+\int_{0}^{-h}\right)(t-s) C_{1}(s) x d s\right],
\end{aligned}
$$

which converges to $\mathbf{C}_{2}\left(C_{1}(t) x-\mathbf{C}_{1} x\right)$ as $h \rightarrow 0$. Thus $y \in \mathcal{D}(A)$ and $A y=$ $C_{1}(t) x-\mathbf{C}_{1} x$, i.e., (1.4) holds.

(d) follows from (c), since

$$
\int_{0}^{t}(t-s) C_{1}(s) x d s \in \mathcal{D}(A), \quad \mathbf{C}_{1} x=2 \lim _{h \rightarrow 0} h^{-2} \int_{0}^{h}(h-s) C_{1}(s) x d s
$$

for any $x \in X$. 
(e) Since $\left\|C_{i}(t)\right\| \leq M e^{\omega|t|}(i=1,2)$ for all $t \in \mathbb{R}$, we define $L_{i}(\lambda) \in$ $B(X)(i=1,2)$ by

$$
L_{i}(\lambda) x \equiv \lambda^{-1} \int_{0}^{\infty} e^{-\lambda t} C_{i}(t) x d t \quad \text { for } x \in X \text { and } \lambda>\omega .
$$

Then, for any $x \in X$, by integration by parts, we have

$$
L_{1}(\lambda) x=\lambda \int_{0}^{\infty} e^{-\lambda t}\left(\int_{0}^{t}(t-s) C_{1}(s) x d s\right) d t \quad \text { for } x \in X \text { and } \lambda>\omega,
$$

which implies that $L_{1}(\lambda) x \in \mathcal{D}(A)$. Since $A$ is closed and $\int_{0}^{t}(t-s) C_{1}(s) x d s \in$ $\mathcal{D}(A)$ for all $t \in \mathbb{R}$ and $x \in X$, we may deduce that

$$
\begin{aligned}
A L_{1}(\lambda) x & =\lambda \int_{0}^{\infty} e^{-\lambda t}\left(A \int_{0}^{t}(t-s) C_{1}(s) x d s\right) d t \\
& =\lambda \int_{0}^{\infty} e^{-\lambda t}\left(C_{1}(t) x-\mathbf{C}_{1} x\right) d t=\lambda^{2} L_{1}(\lambda) x-\mathbf{C}_{1} x
\end{aligned}
$$

for $x \in X$ and $\lambda>\omega$. Thus $\left(\lambda^{2}-A\right) L_{1}(\lambda) x=\mathbf{C}_{1} x$. Similarly, for any $x \in \mathcal{D}(A)$, we have

$$
\begin{aligned}
L_{2}(\lambda) A x & =\lambda^{-1} \int_{0}^{\infty} e^{-\lambda t} C_{2}(t) A x d t=\lambda^{-1} \int_{0}^{\infty} e^{-\lambda t} C_{2}^{\prime \prime}(t) x d t \\
& =\lambda \int_{0}^{\infty} e^{-\lambda t} C_{2}(t) x d t-\mathbf{C}_{2} x
\end{aligned}
$$

and therefore $L_{2}(\lambda)\left(\lambda^{2}-A\right) x=\mathbf{C}_{2} x$, i.e., (1.3) holds. Note that $\mathbf{C}_{2}$ is injective. Thus we see that $\lambda^{2}-A$ is also injective. This and the previous identity $\left(\lambda^{2}-A\right) L_{1}(\lambda) x=\mathbf{C}_{1} x(x \in X)$ imply that $\left(\omega^{2}, \infty\right) \subset \varrho_{\mathbf{C}_{1}}(A)$ and (1.2) holds.

Theorem 1.7. Let $S_{1}(t)=\int_{0}^{t} C_{1}(s) d s$. Suppose that $\left\{C_{1}(t), C_{2}(t)\right\}_{t \in \mathbb{R}}$ is a $\left(\mathbf{C}_{1}, \mathbf{C}_{2}\right)$-regularized cosine existence and uniqueness family generated by $A$. Then

(a) all solutions of (0.1) and (0.2) are unique;

(b) (0.2) is $\mathbf{C}_{1}$-well-posed;

(c) (0.1) is $\mathbf{C}_{1}$-well-posed if $u \in C(\mathbb{R},[\mathcal{D}(A)] \times[\mathcal{D}(A)])$ for every $x, y \in$ $\mathcal{D}(A)$, where $u\left(t, \mathbf{C}_{1} x, \mathbf{C}_{1} y\right)=C_{1}(t) x+S_{1}(t) y$.

Proof. (a) Suppose that $u$ is a solution of (0.1) or (0.2) with $x=y=0$. Then, for all $t, s \in \mathbb{R}$, we have

$$
\frac{d}{d s}\left(\int_{0}^{t-s} C_{2}(r) d r u^{\prime}(s)+C_{2}(t-s) u(s)\right)=0 .
$$


Integrating this equality from 0 to $t$ yields $\mathbf{C}_{2} u(t)=C_{2}(t) u(0)=0$. Note that $\mathbf{C}_{2}$ is injective. It follows that $u(t)=0$ for all $t \in \mathbb{R}$ and this completes the proof.

(b) By Theorem 1.6(a), (c), we see that $v\left(t, \mathbf{C}_{1} x, 0\right)=\int_{0}^{t}(t-s) C_{1}(s) x d s$ is the unique solution of $(0.2)$ with $y=0$ and with $x$ replaced by $C_{1} x$. Hence, the strong continuity of $\left\{C_{1}(t)\right\}_{t \in \mathbb{R}}$ and the Banach-Steinhaus theorem imply that $(0.2)$ is $\mathbf{C}_{1}$-well-posed.

(c) For every $x, y \in \mathcal{D}(A)$, by Theorem 1.6(c), we have

$$
\begin{aligned}
& u\left(t, \mathbf{C}_{1} x, \mathbf{C}_{1} y\right) \\
& \quad=C_{1}(t) x+S_{1}(t) y+A \int_{0}^{t}(t-s)\left(u\left(s, \mathbf{C}_{1} x, \mathbf{C}_{1} y\right)-S_{1}(s) y\right) d s \\
& \quad=\mathbf{C}_{1} x+\mathbf{C}_{1} t y+A \int_{0}^{t}(t-s) u\left(s, \mathbf{C}_{1} x, \mathbf{C}_{1} y\right) d s .
\end{aligned}
$$

Since $A$ is closed, (1.5) and the hypothesis imply that $u\left(t, \mathbf{C}_{1} x, \mathbf{C}_{1} y\right) \in$ $\mathcal{D}(A)$, and $t \mapsto A u\left(t, \mathbf{C}_{1} x, \mathbf{C}_{1} y\right)$ is continuous. Thus, by (a), $u$ is the unique solution of (0.1). From the strong continuity of $C_{1}(t)$, we see that $(0.1)$ is $\mathbf{C}_{1}$-well-posed.

Theorem 1.8. Suppose that $\left\{C_{i}(t)\right\}_{t \in \mathbb{R}} \subseteq B(X)(i=1,2)$ are strongly continuous families. Then the following are equivalent.

(a) $\left\{C_{1}(t), C_{2}(t)\right\}_{t \in \mathbb{R}}$ is a mild $\left(\mathbf{C}_{1}, \mathbf{C}_{2}\right)$-regularized cosine existence and uniqueness family generated by an extension of $A$.

(b) $\int_{0}^{t}(t-s) C_{1}(t) x d s \in \mathcal{D}(A)$ for all $x \in X$ and $t \in \mathbb{R}$, with $C_{1}(t) x-$ $\mathbf{C}_{1} x=A \int_{0}^{t}(t-s) C_{1}(t) x d s, C_{2}(0)=\mathbf{C}_{2}$, and $C_{2}^{\prime \prime}(t) x$ exists and is equal to $C_{2}(t) A x$ for all $x \in \mathcal{D}(A)$ and $t \in \mathbb{R}$.

If $\left\|C_{i}(t)\right\| \leq M e^{\omega|t|}(i=1,2)$ for some $\omega>0$, they are also equivalent to

(c) $t \mapsto C_{1}(t) x$ is a continuous map from $\mathbb{R}$ into $[\mathcal{D}(A)]$ for all $x \in \mathcal{D}(A)$, $\left(\omega^{2}, \infty\right) \subset \varrho_{\mathbf{C}_{1}}(A)$, and (1.2) and (1.3) hold.

Proof. (a) $\Rightarrow(\mathrm{b})$. This is immediate from Theorem 1.6(c) and the definition of the generator.

(b) $\Rightarrow$ (a). We first prove that $\mathbf{C}_{2} C_{1}(t)=C_{2}(t) \mathbf{C}_{1}$ for all $t \in \mathbb{R}$. For any $x \in X$ and $r, t \in \mathbb{R}$,

$$
\begin{aligned}
& \frac{d}{d r}\left[C_{2}(t-r) \int_{0}^{r}(r-u) C_{1}(u) x d u\right] \\
& =-\int_{0}^{t-r} C_{2}(u) C_{1}(r) x d u+\int_{0}^{t-r} C_{2}(u) C_{1} x d u+C_{2}(t-r) \int_{0}^{r} C_{1}(u) x d u .
\end{aligned}
$$


Integrate (1.6) in $r$ from 0 to $t$ to obtain

$$
\begin{aligned}
\mathbf{C}_{2} \int_{0}^{t}(t-u) C_{1}(u) x d u= & -\int_{0}^{t} \int_{0}^{t-r} C_{2}(u) C_{1}(r) x d u d r \\
& +\int_{0}^{t-r} \int_{0}^{t-r} C_{2}(u) C_{1} x d u d r \\
& +\int_{0}^{t r} \int_{0}^{r} C_{2}(t-r) C_{1}(u) x d u d r .
\end{aligned}
$$

Since

$$
\begin{aligned}
\int_{0}^{t} \int_{0}^{r} C_{2}(t-r) C_{1}(u) x d u d r & =\int_{0}^{t} \int_{u}^{t} C_{2}(t-r) C_{1}(u) x d r d u \\
& =\int_{0}^{t} \int_{0}^{t-r} C_{2}(u) C_{1}(r) x d u d r
\end{aligned}
$$

(1.7) implies that

$$
\mathbf{C}_{2} \int_{0}^{t}(t-u) C_{1}(u) x d u=\int_{0}^{t} \int_{0}^{t-r} C_{2}(u) \mathbf{C}_{1} x d u d r .
$$

Differentiating the above equality two times in $t$, we get

$$
\mathbf{C}_{2} C_{1}(t)=C_{2}(t) \mathbf{C}_{1} .
$$

Likewise, we may prove the following fact:

$$
v(t)=A \int_{0}^{t}(t-r) v(r) d r(t \in \mathbb{R}) \Rightarrow v(t)=0(t \in \mathbb{R}),
$$

where $v \in C(\mathbb{R}, X)$. For $s, t \in \mathbb{R}$ and $x \in X$, by (1.4) we have

$$
A \int_{0}^{t}(t-r) C_{2}(s) C_{1}(r) x d r=C_{2}(s) C_{1}(t) x-C_{2}(s) \mathbf{C}_{1} x .
$$

Now (1.4) and a simple computation show that

$$
\begin{aligned}
A \int_{0}^{t}(t-r) \mathbf{C}_{2}\left[C_{1}(r+s)+C_{1}(r-s)\right] x d r & \\
\quad & =\mathbf{C}_{2}\left(C_{1}(t+s)+C_{1}(t-s)\right) x-2 C_{2}(s) C_{1}(t) x .
\end{aligned}
$$

for $s, t \in \mathbb{R}$ and $x \in X$. By (1.8)-(1.11), we have the equality $2 C_{2}(s) C_{1}(t)=$ $\mathbf{C}_{2}\left(C_{1}(t+s)+C_{1}(t-s)\right)$. Thus (a) is proved.

(b) $\Rightarrow(\mathrm{c})$. This is exactly the same as the proof of Theorem 1.6(e). 
(c) $\Rightarrow($ a). Since for all $x \in X$ and $\lambda>\omega$,

$$
\int_{0}^{\infty} e^{-\lambda t} \mathbf{C}_{2} C_{1}(t) x d t=\mathbf{C}_{2} \lambda R\left(\lambda^{2}, A\right) \mathbf{C}_{1} x=\int_{0}^{\infty} e^{-\lambda t} C_{2}(t) \mathbf{C}_{1} x d t,
$$

from the uniqueness of the Laplace transform it follows that $\mathbf{C}_{2} C_{1}(t)=$ $C_{2}(t) \mathbf{C}_{1}(t \in \mathbb{R})$.

To prove that $2 C_{2}(s) C_{1}(t)=\mathbf{C}_{2}\left(C_{1}(t+s)+C_{1}(t-s)\right)$ for all $t, s \in \mathbb{R}$, we shall also use the Laplace transform. For $\mu>\lambda>\omega$ and $x \in X$, we have

$$
\begin{aligned}
\int_{0}^{\infty} & e^{-\mu s} \int_{0}^{\infty} e^{-\lambda t} \mathbf{C}_{2} C_{1}(t+s) x d t d s \\
& =\int_{0}^{\infty} e^{-\mu s} \int_{s}^{\infty} e^{-\lambda(r-s)} \mathbf{C}_{2} C_{1}(r) x d r d s \\
& =\int_{0}^{\infty} e^{-\mu s}\left(e^{\lambda s} \mathbf{C}_{2} \lambda R\left(\lambda^{2}, A\right) \mathbf{C}_{1} x-\int_{0}^{s} e^{-\lambda r} e^{\lambda s} \mathbf{C}_{2} C_{1}(r) x d r\right) d s \\
& =\frac{1}{\mu-\lambda} \mathbf{C}_{2} \lambda R\left(\lambda^{2}, A\right) \mathbf{C}_{1} x-\mathbf{C}_{2} \int_{0}^{\infty} e^{-(\mu-\lambda) s} \int_{0}^{s} e^{-\lambda r} C_{1}(r) x d r d s \\
& =\frac{1}{\mu-\lambda} \mathbf{C}_{2} \lambda R\left(\lambda^{2}, A\right) \mathbf{C}_{1} x-\mathbf{C}_{2} \int_{0}^{\infty} \frac{1}{\mu-\lambda} e^{-(\mu-\lambda) r} e^{-\lambda r} C_{1}(r) x d r \\
& =\frac{\mathbf{C}_{2}}{\mu-\lambda}\left(\lambda R\left(\lambda^{2}, A\right) \mathbf{C}_{1} x-\mu R\left(\mu^{2}, A\right) \mathbf{C}_{1} x\right) \\
& =\frac{\mathbf{C}_{2}}{\mu-\lambda}\left(L_{\lambda} \mathbf{C}_{1} x-L_{\mu} \mathbf{C}_{1} x\right),
\end{aligned}
$$

where $L_{\lambda}=\lambda R\left(\lambda^{2}, A\right)$. Similarly, we obtain

$$
\begin{aligned}
& \int_{0}^{\infty} e^{-\mu s} \int_{0}^{s} e^{-\lambda t} \mathbf{C}_{2} C_{1}(s-t) x d t d s=\frac{\mathbf{C}_{2}}{\lambda+\mu} L_{\mu} \mathbf{C}_{1} x, \\
& \int_{0}^{\infty} e^{-\mu s} \int_{s}^{\infty} e^{-\lambda t} \mathbf{C}_{2} C_{1}(t-s) x d t d s=\frac{\mathbf{C}_{2}}{\lambda+\mu} L_{\lambda} \mathbf{C}_{1} x, \\
& \int_{0}^{\infty} e^{-\mu s} \int_{0}^{\infty} e^{-\lambda t} 2 C_{2}(t) C_{1}(s) x d t d s=2 \mathbf{C}_{2} L_{\lambda} L_{\mu} \mathbf{C}_{1} x .
\end{aligned}
$$

Note that $C_{1}(t)=C_{1}(-t)$. A simple calculation shows that

$$
2 L_{\lambda} L_{\mu}=\frac{1}{\lambda+\mu}\left(L_{\lambda} \mathbf{C}_{1}+L_{\mu} \mathbf{C}_{1}\right)-\frac{1}{\lambda-\mu}\left(L_{\lambda} \mathbf{C}_{1}-L_{\mu} \mathbf{C}_{1}\right)
$$


Combining (1.12)-(1.16) with the uniqueness of the Laplace transform, we see that $2 C_{2}(t) C_{1}(s)=\mathbf{C}_{2}\left(C_{1}(t+s)+C_{1}(t-s)\right)$. Thus $\left\{C_{1}(t), C_{2}(t)\right\}_{t \in \mathbb{R}}$ is a mild $\left(\mathbf{C}_{1}, \mathbf{C}_{2}\right)$-regularized cosine existence and uniqueness family. Also, by (b), $C_{2}^{\prime \prime}(t) x=C_{2}(t) A x$ for all $x \in \mathcal{D}(A)$. This implies that an extension of $A$ generates $\left\{C_{1}(t), C_{2}(t)\right\}_{t \in \mathbb{R}}$.

If we permit only exponentially bounded solutions of (0.1) or (0.2), then a $\mathbf{C}_{1}$-regularized cosine existence family is sufficient to guarantee uniqueness and $\mathbf{C}_{1}$-exponential well-posedness.

Theorem 1.9. Suppose $A$ is closable, $\omega \in \mathbb{R}$. Then there is a mild $C_{1}$ regularized cosine existence family $\left\{C_{1}(t)\right\}_{t \in \mathbb{R}}$ for $A$ such that $\left\|C_{1}(t)\right\| \leq$ $M e^{\omega|t|}$ if and only if

(a) $\left(\omega^{2}, \infty\right) \subseteq \varrho_{\mathbf{C}_{1}}(\bar{A})$, and

(b) (0.2) is $\mathbf{C}_{1}$-exponentially well-posed.

Proof. Suppose that $\left\{C_{1}(t)\right\}_{t \in \mathbb{R}}$ is a mild $\mathbf{C}_{1}$-regularized cosine existence family for $A$. Then, by Definition 1.3, (a) is obvious.

For $x \in X$ and $\lambda>\omega$, we have

$$
\begin{aligned}
\lambda^{2} \int_{0}^{\infty} e^{-\lambda t} A\left(\int_{0}^{t}(t-s)\right. & \left.\left(C_{1}(s) x+S_{1}(s) y\right) d s\right) d t \\
& =\bar{A} \int_{0}^{\infty} e^{-\lambda t} C_{1}(t)\left(x+\frac{1}{\lambda} y\right) d t \\
& =\lambda\left(A-\lambda^{2}+\lambda^{2}\right) R\left(\lambda^{2}, A\right) \mathbf{C}_{1}\left(x+\frac{1}{\lambda} y\right) \\
& =\lambda^{3} R\left(\lambda^{2}, A\right) \mathbf{C}_{1}\left(x+\frac{1}{\lambda} y\right)-\lambda \mathbf{C}_{1}\left(x+\frac{1}{\lambda} y\right) \\
& =\lambda^{2} \int_{0}^{\infty} e^{-\lambda t}\left(C_{1}(t) x+S_{1}(t) y-\left(\mathbf{C}_{1} x+\mathbf{C}_{1} t y\right)\right) d t
\end{aligned}
$$

This implies that

$$
C_{1}(t) x+S_{1}(s) y=A \int_{0}^{t}(t-s)\left(C_{1}(s) x+S_{1}(s) y\right) d s+\mathbf{C}_{1} x+\mathbf{C}_{1} t y
$$

so that $V\left(t, \mathbf{C}_{1} x, \mathbf{C}_{1} y\right)=\int_{0}^{t}(t-s)\left(C_{1}(s) x+S_{1}(s) y\right) d s$ is the desired solution of $(0.2)$.

To prove the uniqueness, suppose that $u^{\prime \prime}(t)=A u(t), u(0)=u^{\prime}(0)=0$, and $u$ is exponentially bounded. Let $\alpha>0$ and $\lambda>\omega$ sufficiently large. A 
direct calculation shows that

$$
\left(\lambda^{2}-\bar{A}\right)\left(\int_{0}^{\alpha} e^{-\lambda t} u(t) d t\right)=\int_{0}^{\alpha}\left(\lambda^{2}-A\right) e^{-\lambda t} u(t) d t=e^{-\lambda \alpha}\left(u^{\prime}(\alpha)+\lambda u(\alpha)\right) .
$$

Since $\bar{A}$ is closed and $u$ is exponentially bounded, we see that $\int_{0}^{\infty} e^{-\lambda t} u(t) d t$ $\in \mathcal{D}(\bar{A})$ and $\left(\lambda^{2}-\bar{A}\right) \int_{0}^{\infty} e^{-\lambda t} u(t) d t=0$ for $\lambda>\omega$. Note that $\lambda^{2}-\bar{A}$ is injective. We find that $u(t) \equiv 0$, as desired. Thus the exponential boundedness of $\left\{C_{1}(t)\right\}_{t \in \mathbb{R}}$ leads to the $\mathbf{C}_{1}$-exponential well-posedness of (0.2).

Suppose that (a) and (b) hold. Let $C_{1}(t) x \equiv \frac{d^{2}}{d t^{2}} V\left(t, \mathbf{C}_{1} x, 0\right)$. Then $\left\{C_{1}(t)\right\}_{t \in \mathbb{R}}$ is a strongly continuous family of bounded operators with $\int_{0}^{t}(t-s) C_{1}(s) x d s \in \mathcal{D}(A)$ and

$$
A\left(\int_{0}^{t}(t-s) C_{1}(s) x d s\right)=C_{1}(t) x-\mathbf{C}_{1} x \quad \text { for } x \in X \text { and } t \in \mathbb{R} .
$$

For $\lambda>\omega$, let $L_{1}(\lambda)=\lambda^{-1} \int_{0}^{\infty} e^{-\lambda t} C_{1}(t) d t$. As in the proof of Theorem 1.6(e), for any $x \in X, L_{1}(\lambda) x \in \mathcal{D}(\bar{A})$, with $\left(\lambda^{2}-\bar{A}\right) L_{1}(\lambda) x=\mathbf{C}_{1} x$. The hypotheses on $A$ now imply that $L_{1}(\lambda) x=\left(\lambda^{2}-A\right)^{-1} \mathbf{C}_{1} x$. Thus our claim holds.

Similarly, we have the following result.

TheOREM 1.10. Suppose that there exists an exponentially bounded $\mathbf{C}_{1}$ regularized cosine existence family for $A$. Then (0.1) is $\mathbf{C}_{1}$-exponentially well-posed.

2. A Hille-Yosida type theorem. In the following, we give HilleYosida characterizations of $\mathbf{C}_{1}$-regularized cosine existence families.

Theorem 2.1. Suppose that $A$ is closed and there exist $M, \omega \geq 0$ such that for all $\lambda>\omega$ and $n \in \mathbb{N}_{0}, \mathcal{R}\left(\mathbf{C}_{1}\right) \subset \mathcal{R}\left((\lambda-A)^{n}\right), \lambda-A$ is injective and

$$
\left\|\left(\lambda\left(\lambda^{2}-A\right)^{-1} \mathbf{C}_{1}\right)^{n}\right\| \leq n ! M(\lambda-\omega)^{-n-1} .
$$

Then for all $\mu>\omega$, there is an exponentially bounded mild $\left(\mu^{2}-A\right)^{-1} \mathbf{C}_{1}$ regularized cosine existence family $\left\{C_{1}(t)\right\}_{t \in \mathbb{R}}$ for $A$.

Proof. Define $f:(0, \infty) \rightarrow B(X)$ by $f(\lambda)=\lambda\left(\lambda^{2}-A\right)^{-1} \mathbf{C}_{1}$. By (2.1) and Corollary 1.2 of [1], there exists a strong continuous family $\{S(t)\}_{t \geq 0}$ satisfying $S(0)=0$ and

$$
\varlimsup_{h \rightarrow 0} \frac{1}{h}\|S(t+h)-S(t)\| \leq M e^{\omega t} \quad \text { for } t \geq 0,
$$

which implies that

$$
\left(\lambda^{2}-A\right)^{-1} \mathbf{C}_{1}=\int_{0}^{\infty} e^{-\lambda t} S(t) d t \quad \text { for } \lambda>\omega \text { and } t \geq 0 .
$$


Set $L_{\lambda}=\lambda\left(\lambda^{2}-A\right)^{-1}$. Then, for $\lambda>\mu>\omega$, from (2.2) we have

$$
\begin{aligned}
& \frac{1}{\mu-\lambda}\left(L_{\lambda} \mathbf{C}_{1}-L_{\mu} \mathbf{C}_{1}\right)=\frac{\lambda-\mu+\mu}{\mu-\lambda} \cdot \frac{1}{\lambda} L_{\lambda} \mathbf{C}_{1}-\frac{1}{\mu-\lambda} L_{\mu} \mathbf{C}_{1} \\
& =-\int_{0}^{\infty} e^{-\lambda t} S(t) d t+\mu \int_{0}^{\infty} \frac{1}{\mu-\lambda} e^{-\lambda \tau} S(\tau) d \tau+\int_{0}^{\infty} e^{-(\lambda-\mu) t} L_{\mu} \mathbf{C}_{1} d t \\
& =\int_{0}^{\infty} e^{-\lambda t}\left(-\mu \int_{0}^{t} e^{\mu(t-\tau)} S(\tau) d \tau-S(t)+e^{\mu t} \mu\left(\mu^{2}-A\right)^{-1} \mathbf{C}_{1}\right) d t
\end{aligned}
$$

Similarly, we obtain

$$
\begin{aligned}
\frac{1}{\lambda+\mu} L_{\mu} & =\int_{0}^{\infty} e^{-\lambda t}\left(e^{-\mu t} \mu\left(\mu^{2}-A\right)^{-1} \mathbf{C}_{1}\right) d t \\
\frac{1}{\lambda+\mu} L_{\lambda} & =\int_{0}^{\infty} e^{-(\lambda+\mu) t}\left(\lambda \int_{0}^{\infty} e^{-\lambda \tau} S(\tau) d \tau\right) d t \\
& =\int_{0}^{\infty} e^{-\lambda t}\left(S(t)-\mu \int_{0}^{t} e^{-\mu(t-\tau)} S(\tau) d \tau\right) d t .
\end{aligned}
$$

By (1.16) and (2.3)-(2.5), we get

$$
\lambda\left(\lambda^{2}-A\right)^{-1}\left(\mu^{2}-A\right)^{-1} \mathbf{C}_{1}=\int_{0}^{\infty} e^{-\lambda t} C_{1}(t) d t \quad \text { for } \lambda>\mu>\omega
$$

where $C_{1}(t)=-\int_{0}^{t} \cosh \mu(t-\tau) S(\tau) d \tau+\cosh (\mu t)\left(\mu^{2}-A\right)^{-1} \mathbf{C}_{1}$. It is obvious that $C_{1}(t)$ is exponentially bounded because $S(t)$ is. We shall simply write $\mathbf{C}=\left(\mu^{2}-A\right)^{-1} \mathbf{C}_{1}$. Then from (2.6) we have

$$
\lambda\left(\lambda^{2}-A\right)^{-1} \mathbf{C}=\int_{0}^{\infty} e^{-\lambda t} C_{1}(t) d t .
$$

As in the proof of [9, Lemma], we obtain $C_{1}(t) x \in \mathcal{D}(A), A C_{1}(t) x=C_{1}(t) A x$ for $x \in \mathcal{D}(A)$ and $t \geq 0$, and

$$
C_{1}(t) x=\mathbf{C} x+\int_{0}^{t}(t-\tau) C_{1}(\tau) A x d \tau \quad \text { for } x \in \mathcal{D}(A) .
$$

Since $A$ is closed, we have $\int_{0}^{t}(t-\tau) C_{1}(\tau) d \tau \in \mathcal{D}(A)$ and $C_{1}(t) x-\mathbf{C} x=$ $A \int_{0}^{t}(t-\tau) C_{1}(\tau) x d \tau$. Thus $\left\{C_{1}(t)\right\}_{t \in \mathbb{R}}$ is a mild $\left(\mu^{2}-A\right)^{-1} \mathbf{C}_{1}$-regularized cosine existence family for $A$.

Corollary 2.2. Suppose that $A$ is closed, $x \in \mathcal{D}(A)$ and there exist $M, \omega>0$ and $\mu>\omega$ such that $\left(\mu^{2}-A\right) x \in \mathcal{R}\left(\left(\lambda^{2}-A\right)^{n}\right)$ for all $\lambda>\omega$ and 
$n \in \mathbb{N}_{0}, \lambda^{2}-A$ is injective for all $\lambda>\omega$ and

$$
\left\|\left(\lambda\left(\lambda^{2}-A\right)^{-1}\right)^{(n)}\left(\mu^{2}-A\right) x\right\| \leq \frac{M n !}{(\lambda-\omega)^{n+1}} \quad \text { for } \lambda>\omega \text { and } n \in \mathbb{N}_{0} .
$$

Then (0.2) has a unique exponentially bounded solution.

The proof of Corollary 2.2 is similar to that of Corollary 5.6 of [5] and we omit it.

3. Examples. In the following, we give a simple example of an operator that does not generate a $C$-regularized cosine function for any $C$. However, it does generate a $\left(\mathbf{C}_{1}, \mathbf{C}_{2}\right)$-regularized cosine existence and uniqueness family. The example is similar to Example 7.1 of [5] (or see [17]).

EXAMPLE 3.1. Let

$$
X \equiv\left\{\text { continuous } f: \mathbb{R} \rightarrow \mathbb{C} \text { satisfying } \lim _{|x| \rightarrow \infty} f(x) e^{x^{2}}=0\right\} .
$$

Then $X$, endowed with the norm $\|f\| \equiv \sup _{x \in \mathbb{R}}\left|f(x) e^{x^{2}}\right|$, is a Banach space. Let

$$
\begin{gathered}
A=\frac{d^{2}}{d x^{2}} \quad(A \text { has maximal domain in } X), \\
\left(\mathbf{C}_{1} f\right)(x)=e^{-x^{2}} f(x), \quad \mathbf{C}_{2} \equiv \mathbf{C}_{1},
\end{gathered}
$$

and let

$$
\begin{aligned}
& \left(C_{1}(t) f\right)(x) \equiv \frac{1}{2}\left(e^{-(x+t)^{2}} f(x+t)+e^{-(x-t)^{2}} f(x-t)\right), \\
& \left(C_{2}(t) f\right)(x) \equiv \frac{1}{2} e^{-x^{2}}(f(x+t)+f(x-t)) .
\end{aligned}
$$

Then it is straightforward to show that $\left(C_{1}(t), C_{2}(t)\right)$ is a $\left(\mathbf{C}_{1}, \mathbf{C}_{2}\right)$-regularized cosine existence and uniqueness family generated by $A$. However, there is no $C$ such that $A$ generates a $C$-regularized cosine function. This is shown by Lemmas 7.2 and 7.3 of [5] and the relation between the regularized semigroup and regularized cosine function (see [18]).

ExAmple 3.2. Suppose that $X_{1}$ and $X_{2}$ are Banach spaces, and $G_{i}(i=$ $1,2)$ is the generator of a strongly continuous cosine function $\left\{S_{i}(t)\right\}_{t \in \mathbb{R}}$ with $\left\|S_{i}(t)\right\|=o\left(e^{\omega_{i}|t|}\right)$ on $X_{i}$ for $i=1,2$. Then there exists an exponentially bounded mild $\mathbf{C}_{1}$-regularized cosine existence family and a $\mathbf{C}_{2}$-regularized cosine uniqueness family on $X_{1} \times X_{2}$ for $A$ where

$$
A=\left[\begin{array}{cc}
G_{1} & 0 \\
0 & G_{2}
\end{array}\right], \quad \mathbf{C}_{1}=\left[\begin{array}{cc}
I & 0 \\
0 & \left(s^{2}-G_{2}\right)^{-1}
\end{array}\right], \quad \mathbf{C}_{2}=\left[\begin{array}{cc}
\left(s^{2}-G_{1}\right)^{-1} & 0 \\
0 & I
\end{array}\right]
$$

for $s>\max \left\{\omega_{1}, \omega_{2}\right\}$. 
Proof. Set

$$
C_{1}(t)=\left[\begin{array}{cc}
S_{1}(t) & 0 \\
0 & S_{2}(t)\left(s^{2}-G_{2}\right)^{-1}
\end{array}\right], \quad C_{2}(t)=\left[\begin{array}{cc}
\left(s^{2}-G_{1}\right)^{-1} S_{1}(t) & 0 \\
0 & S_{2}(t)
\end{array}\right] .
$$

By our assumption, we have

$$
\left(\lambda^{2}-G_{i}\right)^{-1} x=\lambda^{-1} \int_{0}^{\infty} e^{-\lambda t} S_{i}(t) x d t \quad\left(x \in X_{i}, i=1,2\right),
$$

for $\lambda>\max \left\{\omega_{1}, \omega_{2}\right\}$ and $\lambda^{2} \in \varrho\left(G_{1}\right) \cap \varrho\left(G_{2}\right)$. It follows that

$$
\left(\lambda^{2}-A\right)^{-1}=\left[\begin{array}{cc}
\left(\lambda^{2}-G_{1}\right)^{-1} & 0 \\
0 & \left(\lambda^{2}-G_{2}\right)^{-1}
\end{array}\right] \text {. }
$$

Then a calculation shows that

$$
\left(\lambda^{2}-A\right)^{-1} \mathbf{C}_{1} x=\lambda^{-1} \int_{0}^{\infty} e^{-\lambda t} C_{1}(t) x d t \quad\left(x \in X_{1} \times X_{2}\right) .
$$

It is obvious that $\lambda^{2}-A$ is closable and $\lambda^{2}-A$ is injective for $\lambda^{2} \in \varrho\left(G_{1}\right) \cap$ $\varrho\left(G_{2}\right)$. In addition, it is well known that $\int_{0}^{t}(t-s) S_{i}(t) x d s \in \mathcal{D}\left(G_{i}\right)$ for $x \in X_{i}$. Then $\int_{0}^{t}(t-s) C_{1}(s) x d s \in \mathcal{D}(A)$ and $A \int_{0}^{t}(t-s) C_{1}(s) x d s \in C(\mathbb{R}, X)$ for $x \in X_{1} \times X_{2}$ and $t \in \mathbb{R}$. Thus $\left\{C_{1}(t)\right\}_{t \in \mathbb{R}}$ is an exponentially bounded $\mathbf{C}_{1}$-regularized cosine existence family for $A$.

Using the same method, we may prove that $\left\{C_{2}(t)\right\}_{t \in \mathbb{R}}$ is an exponentially bounded $\mathbf{C}_{2}$-regularized cosine uniqueness family for $A$.

\section{References}

[1] W. Arendt, Vector-valued Laplace transforms and Cauchy problems, Israel J. Math. 59 (1987), 327-352.

[2] W. Arendt and H. Kellermann, Integrated solutions of Volterra integro-differential equations and applications, in: Volterra Integro-Differential Equations in Banach Spaces and Applications, Pitman Res. Notes Math. 190, Longman, Harlow, 1989, $21-51$.

[3] C. C. Kuo and S. Y. Shaw, C-cosine functions and the abstract Cauchy problem I, J. Math. Anal. Appl. 210 (1997), 632-646.

[4] -, - C-cosine functions and the abstract Cauchy problem II, ibid. 210 (1997), $647-666$.

[5] R. deLaubenfels, Existence and uniqueness families for the abstract Cauchy problem, J. London Math. Soc. 44 (1991), 310-338.

[6] - Existence Families, Functional Calculi and Evolution Equations, Lecture Notes in Math. 1570, Springer, Berlin, 1994.

[7] R. deLaubenfels, G. Sun and S. Wang, Regularized semigroups, existence families and the abstract Cauchy problem, Differential Integral Equations 8 (1995), 1477-149.

[8] W. Littman, The wave operator and $L^{p}$-norm, J. Math. Mech. 12 (1963), 55-68.

[9] S. I. Miyadera, A generalization of the Hille-Yosida theorem, Proc. Japan Acad. Sect. A 64 (1988), 223-226. 
[10] S. Y. Shaw and Y. C. Li, On n-times integrated $C$-cosine functions, in: Evolution Equations, Dekker, New York, 1994, 393-406.

[11] N. Tanaka, On the exponentially bounded C-cosine family, Gokujutsu Kenkyu (Academic Studies) Math. 37 (1988), 37-44.

[12] C. C. Travis and G. F. Webb, Second order differential equations in Banach space, in: Nonlinear Equations in Abstract Spaces, Academic Press, New York, 1978, 331361.

[13] - , - Compactness, regularity, and uniform continuity properties of strongly continuous cosine families, Houston J. Math. 3 (1977), 555-567.

[14] S. Wang, Mild integrated C-existence families, Studia Math. 112 (1995), 251-266.

[15] S. Wang and Z. Huang, Strongly continuous integrated $C$-cosine operator functions, ibid. 126 (1997), 273-289.

[16] J. Z. Zhang and Q. Zheng, On $\alpha$-times integrated cosine functions, Math. Japon. 50 (1999), 401-408.

[17] Q. Zheng, Integrated cosine functions, Internat. J. Math. Math. Sci. 19 (1996), $575-580$.

[18] Q. Zheng and Y. S. Lei, Exponentially bounded C-cosine functions of operators, J. Systems Sci. Math. Sci. 16 (1996), 242-252 (in Chinese).

Department of Mathematics

Shanghai Normal University

Shanghai 200234, People's Republic of China

E-mail: jizhouzhang@online.sh.cn 